\title{
The Effect of Vasopressors on Perfusion of Gastric Graft after Esophagectomy-Reply to Buise et al.
}

\author{
Stylianos Katsaragakis • Andreas Larentzakis • \\ Panagiotis Drimousis • Dimitrios Theodorou
}

Received: 8 February 2009 /Accepted: 18 February 2009/Published online: 10 March 2009

(C) 2009 The Society for Surgery of the Alimentary Tract

Dear Editor,

We appreciate the comments of Dr. Buise and his team that have extensively studied and published on the effects of several pharmacological agents on splanchnic perfusion.

In our hospital, we use epidural analgesia but especially avoid the use of local anesthetics perioperatively in order to avoid the hypotension that these agents provoke. Regarding the effects of epinephrine on splanchnic perfusion, we have to bear in mind that epinephrine is not only a pure vasoconstrictor but also has a positive inotropic cardiac effect, which increases the cardiac output. As epinephrine is rarely used in the perioperative period, due to several side effects that it has (i.e., tachycardia, coronary artery vasoconstriction), we elected not to study this medication.

It is known that the left gastroepiploic artery is the main vessel in the pigs' gastric greater curvature anatomy. We did preserve the vascular arcade, we used the same technique in all animals, and finally, we had baseline measurements in order to maintain the comparability of our results.

Further measurements and details of the protocol are available but were not found necessary to be included in the presented data.

Yours sincerely

S. Katsaragakis $\cdot$ P. Drimousis $\cdot$ D. Theodorou

1st Department of Propaedeutic Surgery,

"Hippocration" General Hospital of Athens,

Athens Medical School, University of Athens Greece,

Q. Sofias 114 av., 11527,

Athens, Greece

A. Larentzakis $(\square)$

1st Department of Propaedeutic Surgery,

"Hippocration" General Hospital of Athens,

Athens Medical School, University of Athens Greece,

11, 3rd September 1843 str.,

12242 Egaleo, Attiki, Greece

e-mail: alarentz@med.uoa.gr 